\title{
Manipulation Therapy Relieved Pain More Rapidly Than Acupuncture among Lateral Epicondylalgia (Tennis Elbow) Patients: A Randomized Controlled Trial with 8-Week Follow-Up
}

\author{
Chung-Yuan Hsu, ${ }^{1}$ Ko-Hung Lee, ${ }^{2}$ Hsin-Chia Huang, ${ }^{1}$ Zi-Yu Chang, \\ Hsing-Yu Chen, ${ }^{4}$ and Tsung-Hsien Yang ${ }^{1}$ \\ ${ }^{1}$ Division of Chinese Acupuncture and Traumatology, Center of Traditional Chinese Medicine, \\ Taoyuan Chang Gung Memorial Hospital, No. 123, Dinghu Road, Guishan District, Taoyuan 333, Taiwan \\ ${ }^{2}$ Division of Chinese Acupuncture and Traumatology, Center of Traditional Chinese Medicine, Linkou Chang Gung Memorial Hospital, \\ No. 5, Fu-Hsing Street, Guishan District, Taoyuan 333, Taiwan \\ ${ }^{3}$ Division of Chinese Acupuncture and Traumatology, Center of Traditional Chinese Medicine, \\ Keelung Chang Gung Memorial Hospital, No. 222, Maigin Road, Anle District, Keelung 204, Taiwan \\ ${ }^{4}$ Division of Chinese Internal Medicine, Center of Traditional Chinese Medicine, Taoyuan Chang Gung Memorial Hospital, \\ No. 123, Dinghu Road, Guishan District, Taoyuan 333, Taiwan
}

Correspondence should be addressed to Hsin-Chia Huang; ynh714@gmail.com

Received 17 February 2016; Revised 16 March 2016; Accepted 20 March 2016

Academic Editor: Andreas Sandner-Kiesling

Copyright (C) 2016 Chung-Yuan Hsu et al. This is an open access article distributed under the Creative Commons Attribution License, which permits unrestricted use, distribution, and reproduction in any medium, provided the original work is properly cited.

Radial bone adjustment manipulation treatment may be effective to reduce pain rapidly in lateral epicondylalgia patients and the pathological tension in the biceps brachii muscle is highly concerned. To prove this hypothesis, we conducted a randomized controlled trial and included 35 patients with lateral epicondylalgia for more than 2 months. Either manipulation treatment $(n=16)$ or acupuncture $(n=19)$ was given to these patients for 2 weeks and all patients'symptoms were followed up for 8 weeks after treatment. Both groups demonstrated changes in pain VAS score, grip strength, and DASH questionnaire. Lateral epicondylalgia patients who received manipulation treatment felt pain relief sooner than those who had acupuncture treatments during the first few treatments. However, both acupuncture and manipulation are effective, while the difference has no significance at the 8-week follow-up. The trial was registered with Current Controlled Trials ISRCTN81308551 on 5 February 2016.

\section{Introduction}

Lateral epicondylalgia is usually a self-limiting condition; however, symptoms may persist for over 1 year in up to $20 \%$ of patients [1]. Patients with lateral epicondylalgia who fail to improve after nonoperative treatment for 6 months may require additional management for more than 2 years [2]. Current evidence indicates that lateral epicondylalgia is a chronic degenerative disorder with increasing fibroblasts as demonstrated in pathological findings [3-5]. The mechanism recently proposed for pain generation indicates a process of abnormal neural ingrowth that is manifested as peritendinous hypervascularity $[6,7]$.

Although lateral epicondylalgia is highly prevalent, effective treatments are controversial, and combined nonpharmacological treatments are recommended. A recent review of nonsurgical treatments for tennis elbow includes corticosteroid injection, iontophoresis, botulinum toxin A, prolotherapy, platelet-rich plasma or autologous blood injection, bracing, physical therapy, shockwave therapy, and laser therapy; however, the results of these treatments remain nonconclusive [8-10]. Surgical intervention, such as percutaneous 
ultrasonic tenotomy, demonstrated long term effectiveness of pain relief and disability improvement in patients with tennis elbow, but it is invasive and the cost is high [11, 12]. In Traditional Chinese Medicine, manipulation and acupuncture are greatly used in treatments, as well as treating lateral epicondylalgia. Research shows that acupuncture exhibited pain reduction and functional improvement in patients during early follow-up [13]. Similar to manipulation therapy, correcting positional faults by using Mulligan's mobilization with movement (MWM) or cervical spine manipulation is beneficial in pain relief for tennis elbow [14-16]. Up till now, comparative researches between acupuncture and manipulation are still lacking.

We hypothesized that pathological tension in the biceps brachii muscle is related to lateral epicondylalgia according to our clinical experience and physiopathological association. To test this hypothesis, we conducted a clinical trial and investigated the effect of radial bone adjustment therapy on pain relief during rest, daily activity, and work in patients with lateral epicondylalgia. In addition, we evaluated functional improvement and grip force change in this study and all assessments were measured in acupuncture too.

\section{Methods}

2.1. Patients Enrollment. In this single-center, prospective, randomized controlled trial, the enrolled patients with lateral epicondylalgia were either transferred by other doctors in Chinese medicine department or those who saw the information on the bulletin board in Chang Gung Memorial Hospital. These patients were screened for eligibility on the basis of the diagnostic criteria including (1) aggravation of the lateral elbow pain during wrist extension and relief at rest, (2) tenderness of the lateral epicondyle, and (3) positive Cozen's test. However, inclusion criteria were as follows: (1) elbow pain for $>2$ months, (2) unilateral elbow pain, (3) no improvement in the condition despite receiving treatment in previous 4 weeks, and (4) visual analog scale (VAS) score $>30$ millimeters. This study excluded patients who had central or peripheral nervous system diseases, radial nerve entrapment, inflammatory rheumatic disease, gout, or radiocapitellar osteoarthritis, underwent operation for lateral epicondylalgia, or were pregnant. After screening, the patients were randomly assigned computer-generated numbers and, then, divided into an acupuncture group and a manipulation group.

Informed consent was obtained from all participants orally and in writing. The protocol of this trial is approved by the Chang Gung Memorial Foundation, Taipei, Taiwan (IRB number: 99-1544A3).

2.2. Intervention. Patients in the manipulation group received radial bone adjustment to reverse positional fault and relieve the biceps brachii muscle tension. The physician placed one hand on the patient's elbow and the other hand on the wrist. Then, the physician rotated the radial bone internally and extended the biceps brachii muscle simultaneously (Figure 1). The physician performed the manipulation procedure twice in 1 minute with an interval of
30 seconds. The acupuncture group received six acupoints, one Ashi point, LI10, LI11, LU5, LI4, and SJ5, according to a study in Rheumatology published by the Hannover Medical School, Germany [13]. The needle was inserted into the muscle layer and twisted until the de qi sensation was felt. The needle remained in situ for 25 minutes. Both the manipulation and acupuncture groups received the treatments twice per week for 2 weeks.

2.3. Outcome Assessment. Pain relief is the primary outcome of this trial, while improvement in functional impairment and grip strength are regarded as secondary outcomes. For pain assessment, we used the VAS scores [0 (most satisfactory) to 100 (poor)] for recording changes each time before treatment procedure in three states, rest, daily activity, and work situations, from the beginning of the study up to 8 weeks following. For functional impairment, we measured it using the Disability of Arm, Shoulder, and Hand (DASH) questionnaire (developed by the Upper Extremity Collaborative Group) [17, 18]. Patients were evaluated in the beginning of treatment as a baseline and the end of treatment and followed up for 2 and 8 weeks after the end of treatment. In addition, we examined grip strength (pain-free and maximum) by using the Jamar Hand Dynamometer each time before treatment procedure and the follow-up at 2 and 8 weeks after the end of treatment [19].

2.4. Statistical Analysis. Efficacy analyses were performed for all patients assigned to the treatment. The Wilcoxon signed rank test was used to perform within-group comparisons of primary and secondary endpoints. The Mann-Whitney $U$ test was used to perform between-group comparisons of primary and secondary endpoints. All reported $p$ values were two-sided and the value less than 0.05 seems statistically significant. No interim analysis was performed.

\section{Results}

Patients were enrolled from March 2011 to September 2012 and were followed up for 8 weeks after the end of treatment. Of the 53 eligible patients, 18 were excluded. The remaining 35 patients were randomly assigned to the manipulation or acupuncture group. In total, 16 patients were allocated to the manipulation group and 19 patients to the acupuncture group. In the acupuncture group, two of the 19 patients did not complete treatment because of incorrect time coordination. We used the first time data as the baseline and included the last time data in the analyses (Figure 2). In addition, no differences were observed in baseline characteristics between both groups (Table 1).

Improved scores were observed in the pain VAS for both daily activity and during work in both groups (manipulation: daily: baseline: $53.01 \pm 21.70$ versus 8 -week follow-up: 15.97 $\pm 12.62, p<0.001$, work: baseline: $62.13 \pm 16.28$ versus 8 week follow-up: $25.01 \pm 15.74, p<0.001$; acupuncture: daily: baseline: $51.72 \pm 23.04$ versus 8 -week follow-up: $29.68 \pm 21.51$, $p=0.002$, work: baseline: $65.03 \pm 25.48$ versus 8 -week followup: $33.57 \pm 24.05, p<0.001)$. We also found pain VAS for daily activity at the third treatment changed obviously 


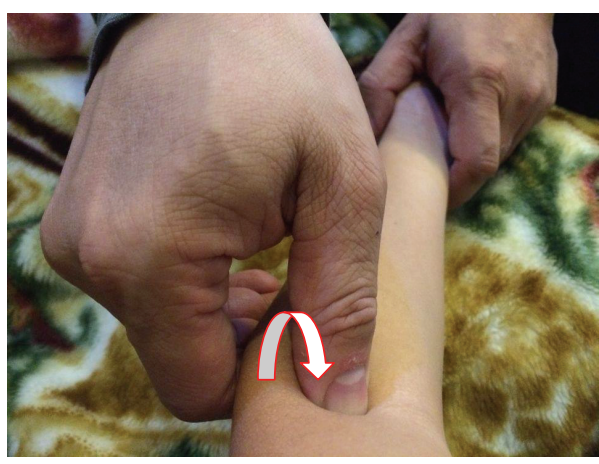

(a)

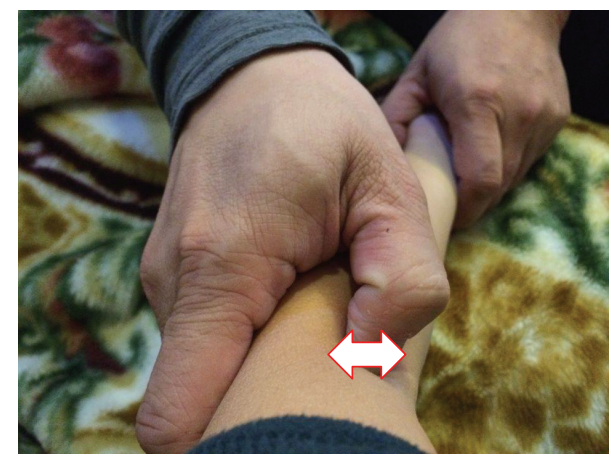

(b)

FIGURE 1: Radial bone adjustment manipulation: (a) rotate the radial bone internally and extend the biceps brachii muscle simultaneously and (b) pluck the contractive biceps brachii muscle to relieve pathological tension.

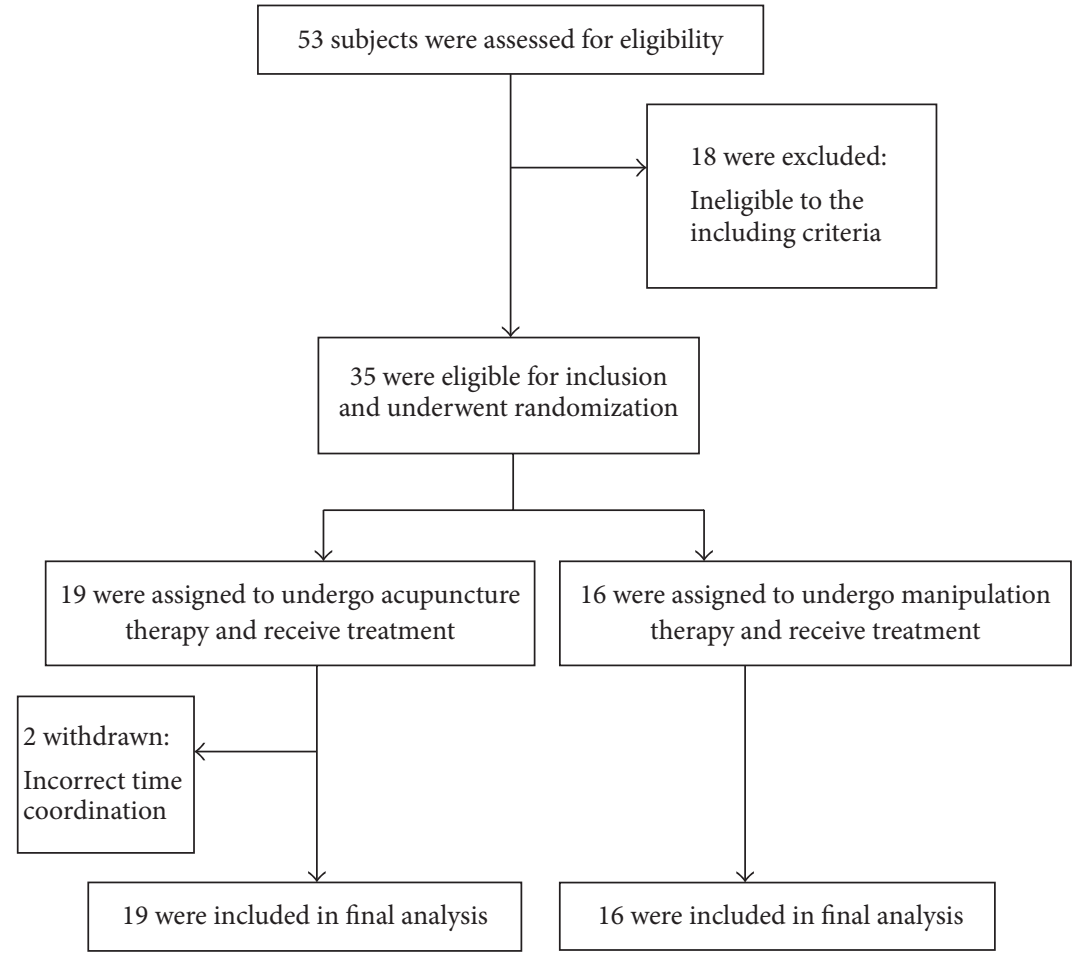

FIGURE 2: Enrollment and randomization of subjects.

in manipulation group, whereas acupuncture group showed no difference at the same time (manipulation: daily: baseline: $53.01 \pm 21.70$ versus the third treatment: $32.39 \pm 20.24, p=$ 0.001 ; acupuncture: daily: baseline: $51.72 \pm 23.04$ versus the third treatment: $43.44 \pm 21.68, p=0.139)$. No significant changes were observed in pain VAS scores at rest in the acupuncture group during 10-week period (baseline: $35.18 \pm$ 24.36 versus 8 -week follow-up: $24.95 \pm 18.90, p=0.165$ ). In contrast, significant changes were observed in pain VAS scores during rest in the manipulation group (baseline: 36.81 \pm 24.94 versus 8 -week follow-up: $13.49 \pm 11.62, p=0.001$; Figure 3).
A significant difference was observed in the DASH questionnaire at the 8-week follow-up in both groups (acupuncture: baseline: $33.56 \pm 17.26$ versus 8 -week follow-up: 20.49 \pm 9.82, $p=0.001$; manipulation: baseline: $31.80 \pm 21.49$ versus 8 -week follow-up: $15.72 \pm 12.31, p<0.001)$ and, more interestingly, the manipulation group exhibited rapid improvement in functional impairment at the end of treatment (baseline: $31.80 \pm 21.49$ versus end of treatment 19.78 $\pm 13.16, p=0.001$; Figure 5). A significant difference was observed in grip strength (pain-free) at the 8-week followup in both groups (acupuncture: baseline: $13.87 \pm 7.87$ versus 8-week follow-up: $19.50 \pm 8.16, p=0.002$; manipulation: 
TABLE 1: Baseline data prior to treatment.

\begin{tabular}{lccc}
\hline & $\begin{array}{c}\text { Manipulation group } \\
n=16\end{array}$ & $\begin{array}{c}\text { Acupuncture group } \\
n=19\end{array}$ & \\
& & & \\
Gender & $11(68.8 \%)$ & $15(78.9 \%)$ & \\
$\quad$ Female & $5(31.2 \%)$ & $(21.1 \%)$ & 0.498 \\
$\quad$ Male & $44.81 \pm 7.30$ & $45.89 \pm 5.99$ & 0.619 \\
Age & $22.83 \pm 3.21$ & $23.41 \pm 2.75$ & 0.380 \\
BMI $\left(\mathrm{kg} / \mathrm{m}^{2}\right)$ & $66.91 \pm 136.09$ & $134.89 \pm 258.09$ & 0.079 \\
Pain duration (week) & $31.80 \pm 21.49$ & $33.56 \pm 17.26$ & 0.619 \\
DASH score (0-100) & & $35.18 \pm 24.36$ & 0.881 \\
Pain VAS (0-100, mm) & $36.81 \pm 24.94$ & $51.72 \pm 23.04$ & 0.584 \\
Rest & $53.01 \pm 21.70$ & $65.03 \pm 25.48$ & $13.37 \pm 7.87$ \\
$\quad$ Daily activity & $62.13 \pm 16.28$ & $19.20 \pm 9.03$ \\
$\quad$ Work & $15.20 \pm 10.92$ & 0.540 \\
Pain-free grip strength $(\mathrm{kg})$ & $21.79 \pm 12.10$ & 0.631 \\
Maximum grip strength $(\mathrm{kg})$ & & 0.497 \\
\hline
\end{tabular}

No differences in baseline characteristics among the study participants.

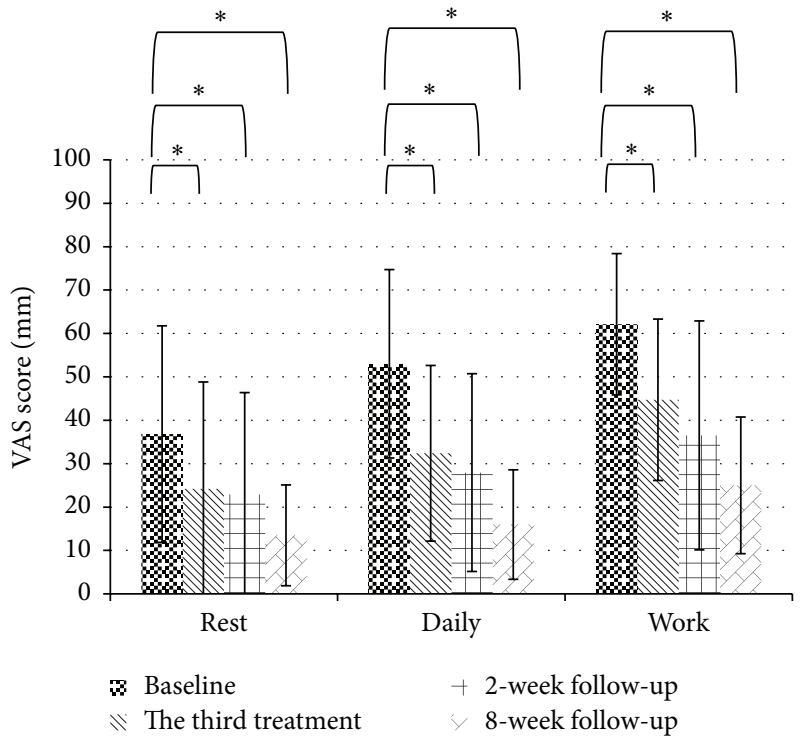

(a)

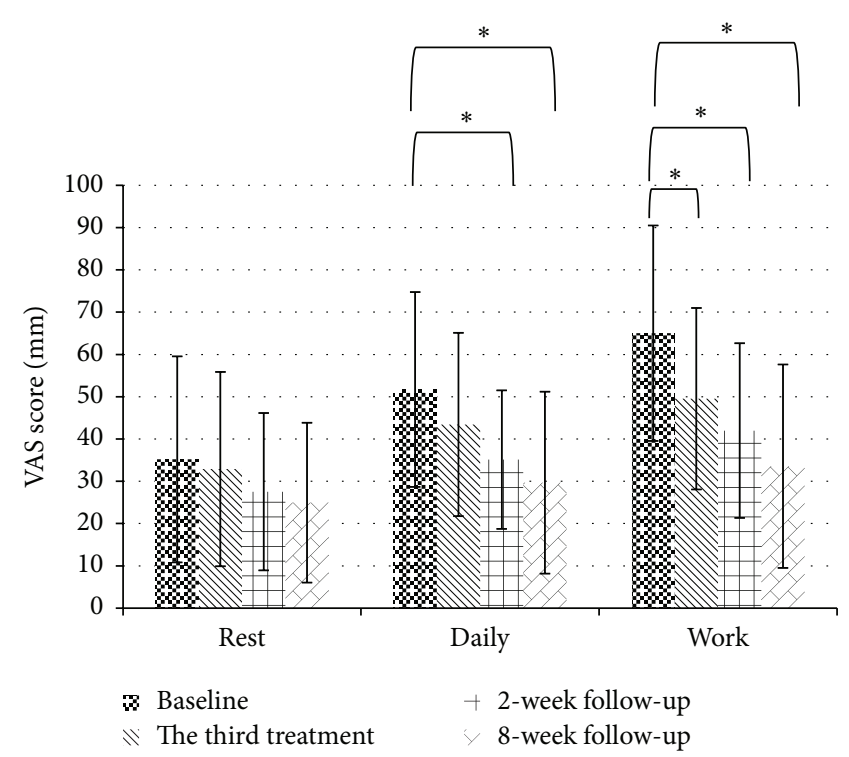

(b)

FIGURE 3: Mean pain VAS scores during the 10-week study period. (a) Manipulation group. (b) Acupuncture group. $\left({ }^{*}\right.$ Comparisons to baseline, $p$ value $<0.05$.)

baseline: $15.20 \pm 10.92$ versus 8 -week follow-up: $19.52 \pm 9.67$, $p=0.015$ ), whereas a significant difference was observed only in the acupuncture group for grip strength (maximum) (acupuncture: baseline: $19.21 \pm 9.06$, versus 8-week follow-up: $23.17 \pm 8.85, p=0.005$, versus manipulation: baseline: 21.79 \pm 12.10 , versus 8 -week follow-up: $24.26 \pm 10.70, p=0.163$; Figure 4).

No serious adverse event was observed in both groups. Light hemorrhage or hematoma was the most common adverse event in the acupuncture group and was treated by compression with an aseptic oral cotton swab. Local pain occurred in the manipulation group; however, it was relieved spontaneously after treatment.

\section{Discussion}

This is the first prospective, comparative, randomized controlled trial among lateral epicondylalgia patients who respond poorly to conventional western medicine treatment. Both manipulation and acupuncture serve as potential efficient treatment for these patients, and manipulation seems more effective during first few treatments than acupuncture in pain relief.

According to the studies on Mulligan's mobilization with movement (MWM), positional faults and subluxations are the main causes of pain, and reversal of positional faults can rapidly alleviate pain and improve functional 


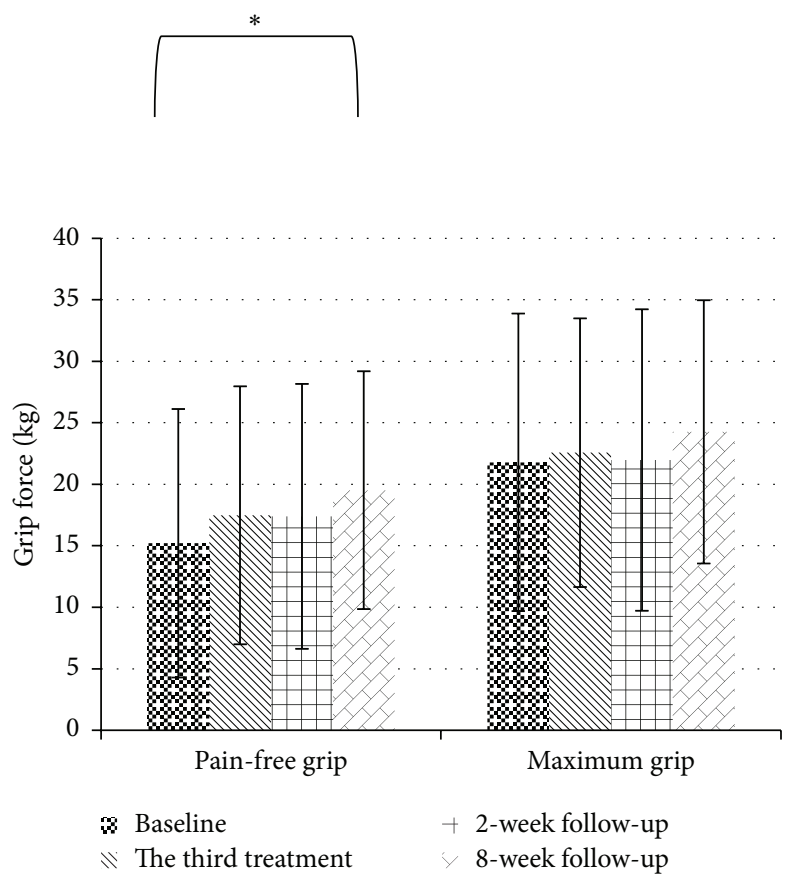

(a)

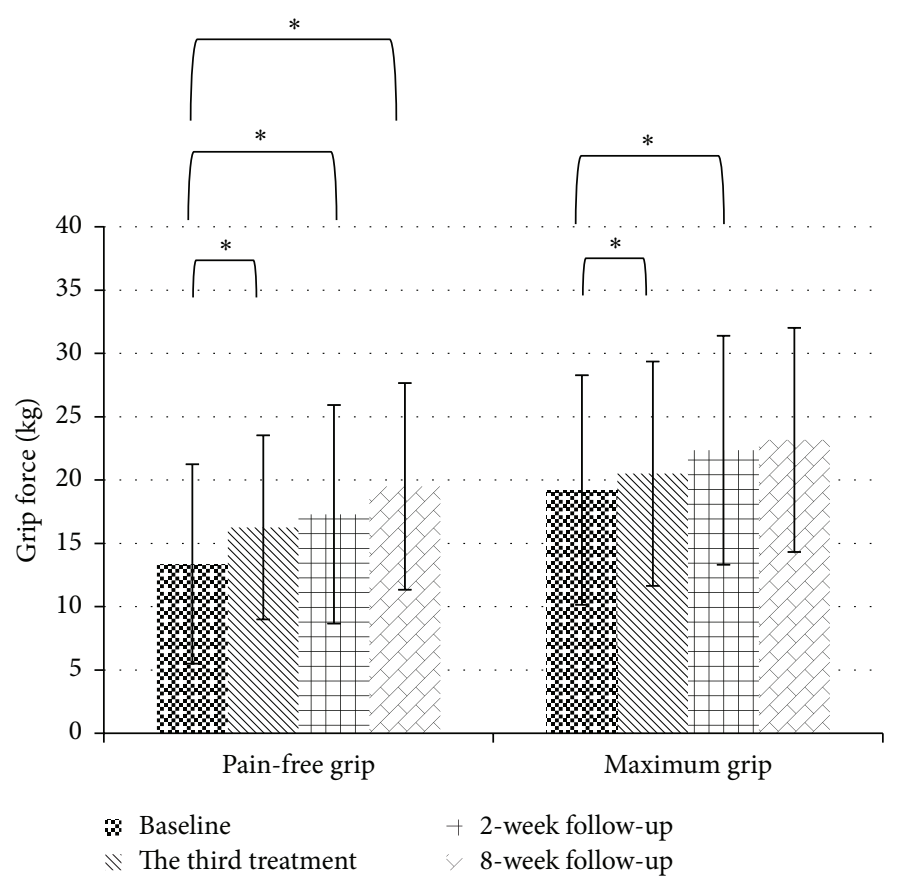

(b)

Figure 4: Mean grip strength during the 10-week study period. (a) Manipulation group. (b) Acupuncture group. ( ${ }^{*}$ Comparisons to baseline, $p$ value $<0.05$.

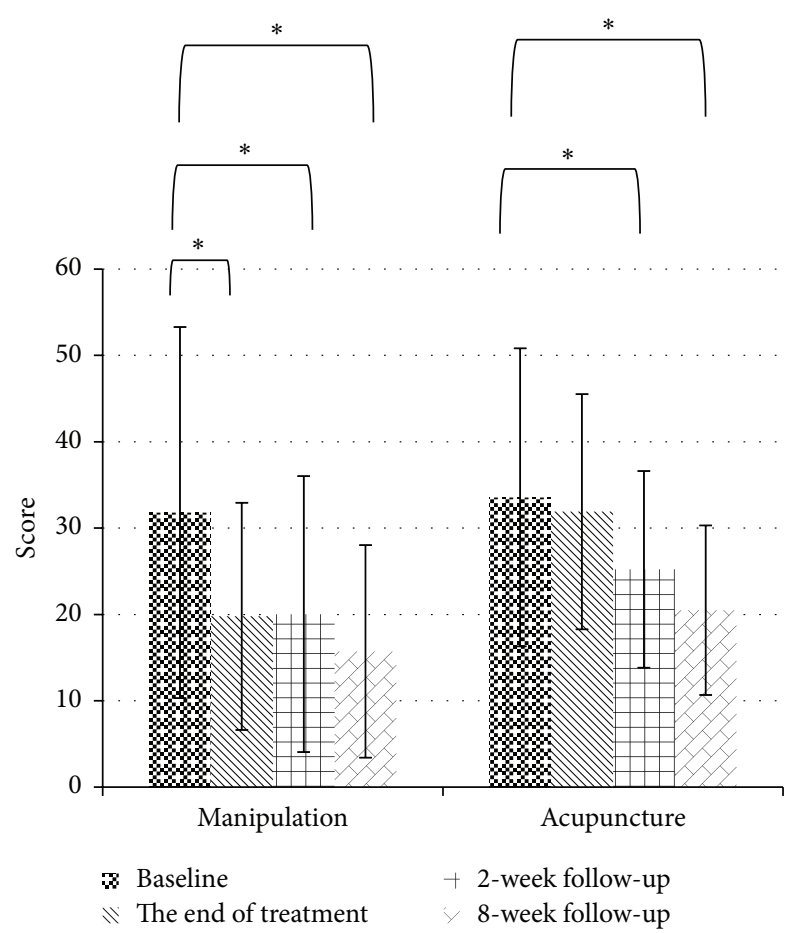

Figure 5: Mean DASH during the 10-week study period. $\left({ }^{*}\right.$ Comparisons to baseline, $p$ value $<0.05$.)

impairment $[3,20]$. The reason to the result may come from direct force that was applied to correct positional faults, whereas acupuncture attempts to release the tension in the extensor muscles. The high tension in the extensor muscles increases the humeroradial joint shear force and may lead to positional faults or subluxations under unstable conditions $[21,22]$. However, decreasing shear force on the humeroradial joint by smoothing extensor muscles tension will gradually reverse positional faults of radial bone.

The biceps brachii muscle is the major supinator of elbow and it is attached to the radial tuberosity. Pathological tension of biceps brachii muscle presented by muscle spasm causing lateral rotation of the radial bone passively according to physiopathology in patients with lateral epicondylalgia [23]. The supinated radial bone aggravates the tension to the lateral collateral ligament and the annular ligament which the deep extensor carpi radialis brevis tendon merges with directly and indirectly [4]. Thence, internal rotation of radial bone in our manipulation can reverse the positional faults and the least biceps brachii muscle tension is also in this position. Consequently, we pluck the contractive biceps brachii muscle to relieve pathological tension and it is necessary to maintain the efficacy, whereas it is lacking in acupuncture. Therefore, manipulation for lateral epicondylalgia is more prominent to acupuncture in pain relief.

The previous studies showed the pain reduction mechanism using manipulative therapy related to spinal cord hyperexcitability, especially sensory hypersensitivity exhibited in nociceptive pathway by peripheral afferent stimulation [24]. In addition, central sensitization by decreasing of pressure pain threshold in generalized deep tissue in lateral epicondylalgia was demonstrated [25]. Therefore, the potential of pain reduction of radial bone adjustment manipulation may be related to nonopioid-mediated hypoalgesia in which 
the effect of peripheral mobilization treatment technique is not inhibited by naloxone in lateral epicondylalgia $[26,27]$.

Acupuncture is also a convenient and economical option for treating lateral epicondylalgia. In a trial conducted in the Hannover Medical School, Germany, pain reduction and functional improvement of the arm were observed in early follow-up after the acupuncture treatment [13]. The same methods were used in this study and the results are consistent with their results.

Brain image with fMRI supports that traditional acupuncture point stimulation activates different response from stimulation at other points on same spinal segment [28]. Moreover, releasing neurotransmitter of opioids in central nervous system by acupuncture is demonstrated in previous studies [29-31]. As a result, the potential mechanism of pain relief may be associated with the activation of the hypothalamus causing opioid peptides alteration and represent descending inhibitory pathway [32,33].

Acupuncture is more impressive than manipulation in grip strength changes, especially in maximum grip strength. Since collagen type I is the major tendon structure component, the possible reason may be that acupuncture stimuli increase synthesis of collagen type I concentration and reorganize it by anti-inflammatory and mechanotransduction molecular pathways [34]. However, the result exhibited painfree grip changes at 8 -week follow-up in manipulation. The possible reasons may come from decreased pathological tension in the high extensor muscles, improved circulation, accelerated cell regeneration, and reversed degeneration process after a few weeks.

Both groups demonstrated well changes in DASH questionnaire and it presented improvement of quality and quantity of life and functional impairment. In our study, the patients who received manipulation treatment showed faster improvement than acupuncture group during treatment period, but both were effective at the 8-week followup. The possible reason may be that the subjective sense of our patients about quality of daily activity is more related to painful sensation than the real grip strength.

Compared to other invasive reports, the total cost of the procedure in our study in Taiwan is approximately NT $\$ 4000$ (US\$120) and is covered under the National Health Insurance. This technique provides definitive intervention for recalcitrant tendinopathy without any drug or surgery, which would require huge cost and resources.

Additional studies using ultrasonographic assessment for demonstrating the changes in the histology and pathology after these treatments are still warranted. Furthermore, other opioid peptides would not be antagonized by naloxone [26] and radial bone adjustment manipulation induced hypoalgesia mechanism would be clarified in further studies.

\section{Conclusion}

The novel manipulation technique improved pain in patients with lateral epicondylalgia (tennis elbow) during the first few treatments till 8-week follow-up.

\section{Competing Interests}

The authors declare that they have no competing interests.

\section{Authors' Contributions}

Ko-Hung Lee and Hsin-Chia Huang contributed to the conception and design of the study. Chung-Yuan Hsu participated in the literature search, writing of the paper, and editing of the paper. Zi-Yu Chang and Tsung-Hsien Yang acquired and analyzed the data. Hsing-Yu Chen proofread the paper. All the authors have read and approved the final submitted paper. Chung-Yuan Hsu and Ko-Hung Lee contributed equally to this paper.

\section{References}

[1] L. Bisset, B. Coombes, and B. Vicenzino, “Tennis elbow," BMJ Clinical Evidence, In press.

[2] R. P. Nirschl, "The epidemiology and health care burden of tennis elbow: a population-based study," Annals of Translational Medicine, vol. 3, no. 10, p. 133, 2015.

[3] C. R. Herd and B. B. Meserve, "A systematic review of the effectiveness of manipulative therapy in treating lateral epicondylalgia," Journal of Manual and Manipulative Therapy, vol. 16, no. 4, pp. 225-237, 2008.

[4] B. K. Coombes, L. Bisset, and B. Vicenzino, "A new integrative model of lateral epicondylalgia," British Journal of Sports Medicine, vol. 43, no. 4, pp. 252-258, 2009.

[5] Z. Ahmad, N. Siddiqui, S. S. Malik, M. Abdus-Samee, G. Tytherleigh-Strong, and N. Rushton, "Lateral epicondylitis: a review of pathology and management," Bone and Joint Journal $B$, vol. 95, no. 9, pp. 1158-1164, 2013.

[6] H. Alfredson, L. Öhberg, and S. Forsgren, "Is vasculoneural ingrowth the cause of pain in chronic Achilles tendinosis? An investigation using ultrasonography and colour Doppler, immunohistochemistry, and diagnostic injections," Knee Surgery, Sports Traumatology, Arthroscopy, vol. 11, no. 5, pp. 334-338, 2003.

[7] Ø. Lian, J. Dahl, P. W. Ackermann, F. Frihagen, L. Engebretsen, and R. Bahr, "Pronociceptive and antinociceptive neuromediators in patellar tendinopathy," The American Journal of Sports Medicine, vol. 34, no. 11, pp. 1801-1808, 2006.

[8] S. E. G. Sims, K. Miller, J. C. Elfar, and W. C. Hammert, "Nonsurgical treatment of lateral epicondylitis: a systematic review of randomized controlled trials," Hand, vol. 9, no. 4, pp. 419-446, 2014.

[9] M. Faes, B. van den Akker, J. A. de Lint, J. G. M. Kooloos, and M. T. E. Hopman, "Dynamic extensor brace for lateral epicondylitis," Clinical Orthopaedics and Related Research, vol. 442, pp. 149-157, 2006.

[10] R. Tosti, J. Jennings, and J. M. Sewards, "Lateral epicondylitis of the elbow," American Journal of Medicine, vol. 126, no. 4, pp. 357.e1-357.e6, 2013.

[11] C. Seng, P. C. Mohan, S. B. Koh et al., "Ultrasonic percutaneous tenotomy for recalcitrant lateral elbow tendinopathy: sustainability and sonographic progression at 3 years," The American Journal of Sports Medicine, vol. 44, no. 2, pp. 504-510, 2016.

[12] M. Y. Lo and M. R. Safran, "Surgical treatment of lateral epicondylitis: a systematic review," Clinical Orthopaedics and Related Research, no. 463, pp. 98-106, 2007. 
[13] M. Fink, E. Wolkenstein, M. Karst, and A. Gehrke, "Acupuncture in chronic epicondylitis: a randomized controlled trial," Rheumatology, vol. 41, no. 2, pp. 205-209, 2002.

[14] B. Vicenzino, A. Paungmali, and P. Teys, "Mulligan's mobilization-with-movement, positional faults and pain relief: current concepts from a critical review of literature," Manual Therapy, vol. 12, no. 2, pp. 98-108, 2007.

[15] J. Fernández-Carnero, J. A. Cleland, and R. L. T. Arbizu, "Examination of motor and hypoalgesic effects of cervical vs thoracic spine manipulation in patients with lateral epicondylalgia: a clinical trial," Journal of Manipulative and Physiological Therapeutics, vol. 34, no. 7, pp. 432-440, 2011.

[16] B. Vicenzino, J. A. Cleland, and L. Bisset, "Joint manipulation in the management of lateral epicondylalgia: a clinical commentary," Journal of Manual and Manipulative Therapy, vol. 15, no. 1, pp. 50-56, 2007.

[17] P. L. Hudak, P. C. Amadio, and C. Bombardier, "Development of an upper extremity outcome measure: the DASH (disabilities of the arm, shoulder and hand) [corrected]. The Upper Extremity Collaborative Group (UECG)," American Journal of Industrial Medicine, vol. 29, no. 6, pp. 602-608, 1996.

[18] O. Alizadehkhaiyat, A. C. Fisher, G. J. Kemp, and S. P. Frostick, "Pain, functional disability, and psychologic status in tennis elbow," Clinical Journal of Pain, vol. 23, no. 6, pp. 482-489, 2007.

[19] J. V. Bellace, D. Healy, M. P. Besser, T. Byron, and L. Hohman, "Validity of the Dexter Evaluation System's Jamar dynamometer attachment for assessment of hand grip strength in a normal population," Journal of Hand Therapy, vol. 13, no. 1, pp. 46-51, 2000.

[20] L. Exelby, "Peripheral mobilisations with movement," Manual Therapy, vol. 1, no. 3, pp. 118-126, 1996.

[21] H. C. Hsu, H. C. Huang, and K. H. Lee, "Manipulation of tennis elbow: a case report," Taipei Journal of Chinese Medicine, vol. 20, no. 1, pp. 67-74, 2014.

[22] D. M. Kalainov and M. S. Cohen, "Posterolateral rotatory instability of the elbow in association with lateral epicondylitis: a report of three cases," Journal of Bone and Joint Surgery A, vol. 87, no. 5, pp. 1120-1125, 2005.

[23] D. A. Neumann, Kinesiology of the Musculoskeletal System: Foundations for Rehabilitation, Elsevier Health Sciences, 2013.

[24] E. C. W. Lim, M. Sterling, A. Pedler, B. K. Coombes, and B. Vicenzino, "Evidence of spinal cord hyperexcitability as measured with nociceptive flexion reflex (NFR) threshold in chronic lateral epicondylalgia with or without a positive neurodynamic test," Journal of Pain, vol. 13, no. 7, pp. 676-684, 2012.

[25] J. Fernández-Carnero, C. Fernández-de-Las-Peñas, A. I. de la Llave-Rincón, H.-Y. Ge, and L. Arendt-Nielsen, "Widespread mechanical pain hypersensitivity as sign of central sensitization in unilateral epicondylalgia: a blinded, controlled study," Clinical Journal of Pain, vol. 25, no. 7, pp. 555-561, 2009.

[26] A. Paungmali, S. O'Leary, T. Souvlis, and B. Vicenzino, "Naloxone fails to antagonize initial hypoalgesic effect of a manual therapy treatment for lateral epicondylalgia," Journal of Manipulative and Physiological Therapeutics, vol. 27, no. 3, pp. 180-185, 2004.

[27] J. Fernández-Carnero, C. Fernández-De-Las-Peñas, A. I. de la Llave-Rincón, H.-Y. Ge, and L. Arendt-Nielsen, "Bilateral myofascial trigger points in the forearm muscles in patients with chronic unilateral lateral epicondylalgia: a blinded, controlled study," Clinical Journal of Pain, vol. 24, no. 9, pp. 802-807, 2008.

[28] W.-T. Zhang, Z. Jin, F. Luo, L. Zhang, Y.-W. Zeng, and J.-S. Han, "Evidence from brain imaging with fMRI supporting functional specificity of acupoints in humans," Neuroscience Letters, vol. 354, no. 1, pp. 50-53, 2004.

[29] J.-S. Han, "Acupuncture: neuropeptide release produced by electrical stimulation of different frequencies," Trends in Neurosciences, vol. 26, no. 1, pp. 17-22, 2003.

[30] D. J. Mayer, D. D. Price, and A. Rafii, "Antagonism of acupuncture analgesia in man by the narcotic antagonist naloxone," Brain Research, vol. 121, no. 2, pp. 368-372, 1977.

[31] V. Clement-Jones, L. McLoughlin, S. Tomlin, G. M. Besser, L. H. Rees, and H. L. Wen, "Increased $\beta$-endorphin but not metenkephalin levels in human cerebrospinal fluid after acupuncture for recurrent pain," The Lancet, vol. 2, no. 8201, pp. 946-948, 1980.

[32] L. Lin, N. Skakavac, X. Lin et al., "Acupuncture-induced analgesia: the role of microglial inhibition," Cell Transplantation, vol. 25, no. 4, pp. 621-628, 2016.

[33] K. J. Cheng, "Neurobiological mechanisms of acupuncture for some common illnesses: a clinician's perspective," Journal of Acupuncture and Meridian Studies, vol. 7, no. 3, pp. 105-114, 2014.

[34] S. Almeida Mdos, R. Guerra Fda, L. P. de Oliveira, C. P. Vieira, and E. R. Pimentel, "A hypothesis for the anti-inflammatory and mechanotransduction molecular mechanisms underlying acupuncture tendon healing," Acupuncture in Medicine, vol. 32, no. 2, pp. 178-182, 2014. 


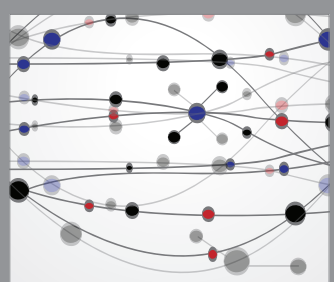

The Scientific World Journal
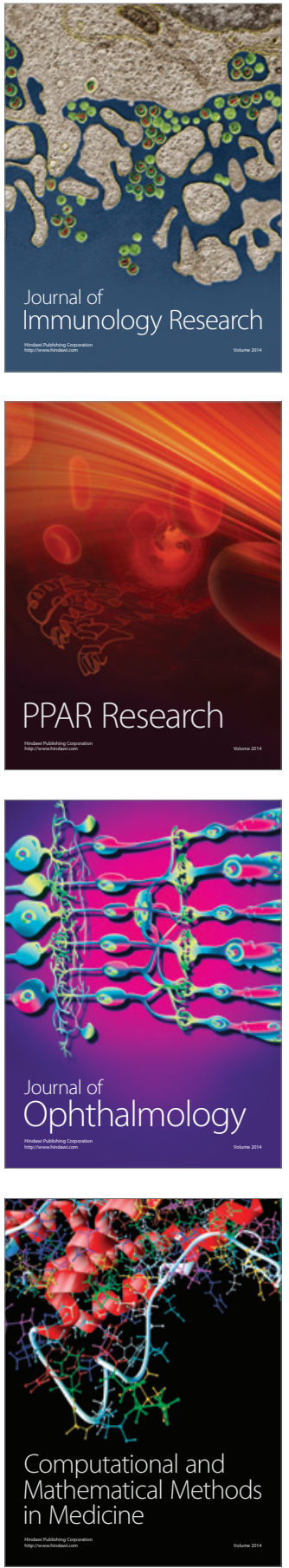

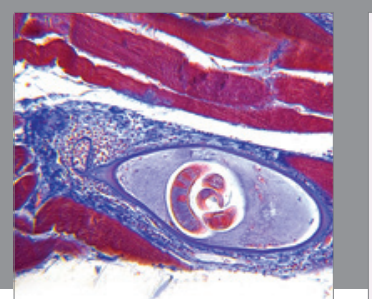

Gastroenterology Research and Practice

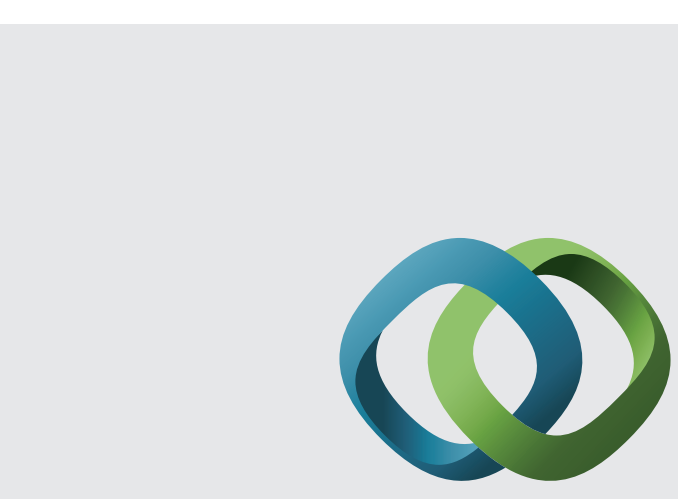

\section{Hindawi}

Submit your manuscripts at

http://www.hindawi.com
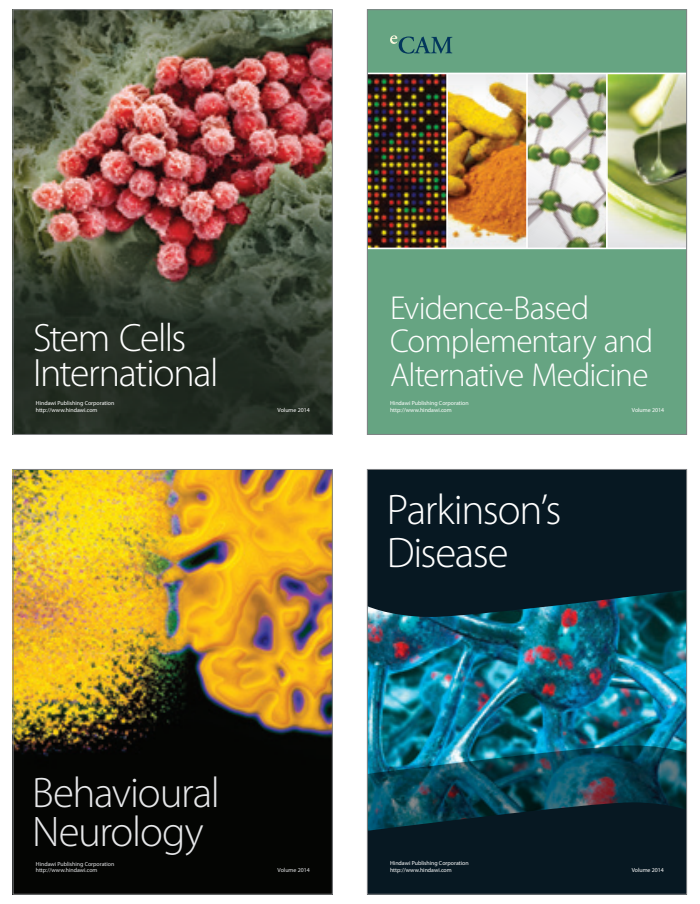
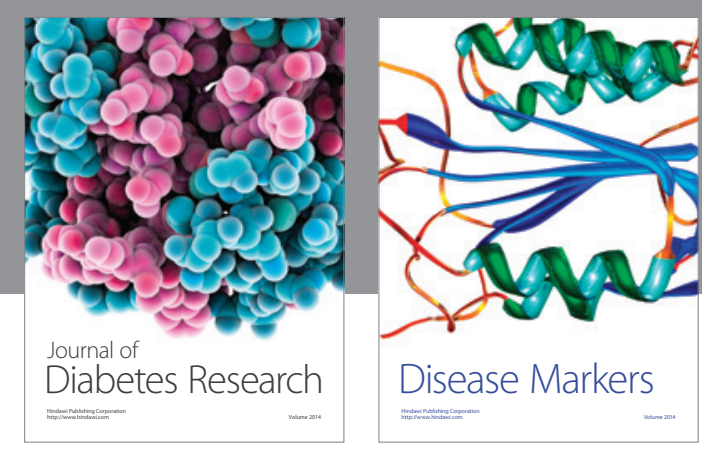

Disease Markers
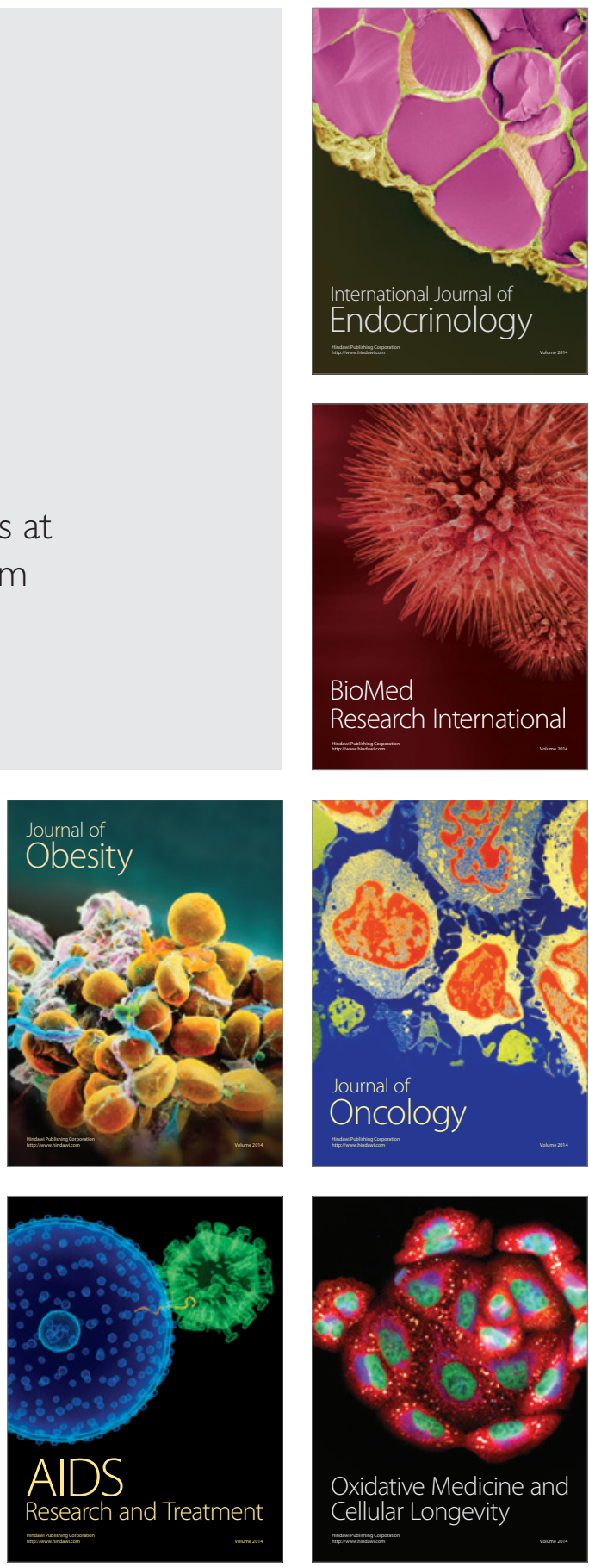